\title{
Adenocarcinoma of the lung with concomitant ALK fusion gene and EGFR gene mutation: A case report and literature review
}

\author{
TAO FAN ${ }^{1}$, YING-JIE SONG ${ }^{2}$ and XIU-LI LIU ${ }^{1}$ \\ Departments of ${ }^{1}$ Oncology and ${ }^{2}$ General Surgery, First People's Hospital of Yichang, \\ People's Hospital of Three Gorges University, Yichang, Hubei 443000, P.R. China
}

Received September 2, 2015; Accepted October 16, 2015

DOI: $10.3892 / \mathrm{mco} .2015 .684$

\begin{abstract}
Targeted therapy is currently a very popular approach to cancer treatment. Personalized targeted therapy of non-small-cell lung cancer (NSCLC) is based on the mutation status of epithelial growth factor receptor (EGFR), Kirsten rat sarcoma viral oncogene homolog, B-Raf proto-oncogene and anaplastic lymphoma kinase (ALK) fusion gene, with different mutations requiring different treatment. Herein, we present a rare case of adenocarcinoma of the lung harboring EGFR- and ALK-activating mutations simultaneously. The patient is currently on pemetrexed and carboplatin chemotherapy and faring well; follow-up will be continued. It is recommended that the biological behavior of cancers with coexisting genetic mutations and their clinicopathological characteristics are more closely investigated in future studies, in order to determine the sensitivity of such cancers to targeted drugs and select the optimal agent.
\end{abstract}

\section{Introduction}

Non-small lung cancer (NSCLC) accounts for $~ 80-85 \%$ of all lung carcinomas. It includes squamous cell carcinoma, adenocarcinoma, large cell carcinoma,compared with small cell carcinoma, the growth of cancer cells divide more slowly, diffusion transfer relatively late. Approximately $90 \%$ of NSCLC cases are caused by smoking and the use of tobacco products. However, other factors such as radon gas, asbestos, air pollution exposures, and chronic infections can contribute to lung carcinogenesis. Treatment options for NSCLC include surgery, radiation therapy, chemotherapy, and targeted therapy (1).

Correspondence to: Ms. Ying-Jie Song, Department of General Surgery, First People's Hospital of Yichang, People's Hospital of Three Gorges University, 2 Jiefang Road, Yichang, Hubei 443000, P.R. China

E-mail: 123525090@qq.com

Key words: adenocarcinoma, anaplastic lymphoma kinase fusion gene, epithelial growth factor receptor gene mutation
Targeted therapy is currently a very popular approach to cancer treatment. Personalized targeted therapy of NSCLC is based on the mutation status of epithelial growth factor receptor (EGFR), Kirsten rat sarcoma viral oncogene homolog (K-Ras), B-Raf proto-oncogene (BRAF) and anaplastic lymphoma kinase (ALK) fusion gene, with different mutations requiring different treatment. Herein, we present a rare case of adenocarcinoma of the lung harboring EGFR- and ALK-activating mutations simultaneously.

\section{Case report}

A 63-year-old female patient was admitted to the First People's Hospital of Yichang (Yichang, China) in June, 2015 due to the presence of productive cough for 3 months. The patient provided written informed consent for the publication of her case details. The patient was a non-smoker and had a history of benign breast tumor resection and allergy to penicillin 10 years earlier. The physical examination did not reveal any significant abnormalities. On chest X-ray there was an abnormal shadow in the lower field of the left lung. The positron emission tomography-computed tomography scan revealed a highly metabolic mass lesion in the left lower lobe, measuring $5.0 \mathrm{~cm}$ in the largest dimension in the $\mathrm{S} 3$ segment. The results of the head magnetic resonance imaging scan, isotope bone scan, abdominal ultrasound, bronchoscopy and laboratory tests were normal. The patient's blood type was AB, $\mathrm{RhD}^{+}$. A transbronchial lung biopsy (TBLB) was conducted and the pathological diagnosis of the TBLB specimen was invasive adenocarcinoma (cT2N0M0). As there were no surgical contraindications, the patient underwent left lower lobe resection and hilar mediastinal lymph node dissection. The final pathology results revealed that the nodule in the left S3 segment was invasive adenocarcinoma (80\% adherent and $20 \%$ papillary growth pattern), and invading the wall of the trachea or bronchus; the bronchial stump was cancer-free and there was no tumor invasion of the lymph nodes $(0 / 22)$ (pT2N0M0). A fresh surgical specimen was sent for molecular analysis (Fig. 1A), confirming that the tumor harbored an EGFR mutation (19-del). An ALK (D5F3+) rearrangement mutation was also detected by fluorescence polymerase chain reaction (Fig. 1B and C). The patient is currently on pemetrexed and carboplatin chemotherapy and faring well. Based on the presence of an ALK rearrangement, crizotinib administra- 

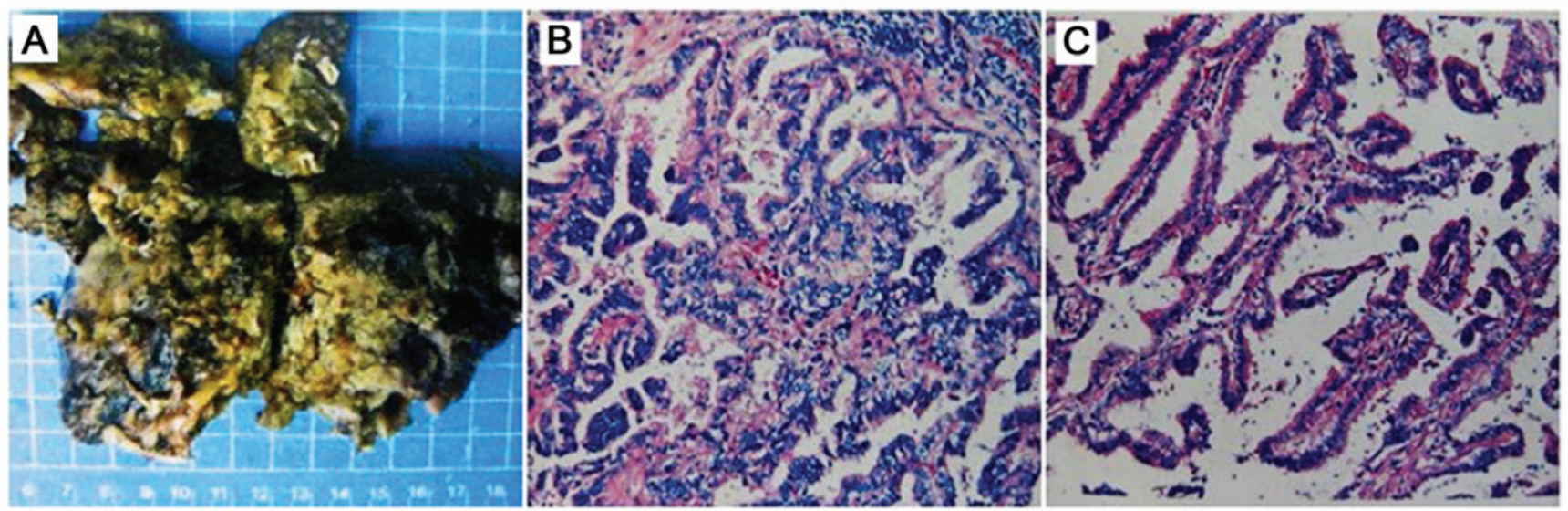

Figure 1. Invasive adenocarcinoma (80\% adherent and 20\% papillary growth pattern. (A) Photograph of the excised specimen, measuring 13x7x6 cm; (B) haematoxylin and eosin staining, magnification, x100; and (C) immunohistochemical staining for ALK (D5F3+), magnification, x400.

tion was considered. However, as the tumor also harbored an EGFR mutation, the EGFR-tyrosine kinase inhibitors (TKIs) gefitinib and erlotinib were selected.

\section{Discussion}

We herein report an unusual case of a female patient with adenocarcinoma of the lung harboring EGFR (19-del) and ALK rearrangement. To the best of our knowledge, Yang et al (2) reported a case of a male patient with adenocarcinoma of the lung harboring an EGFR mutation (19-del), ALK rearrangement and a BRAF (V600E) mutation in October, 2014. Recent advances in molecular status analyses of lung adenocarcinoma specimens have revealed that activating mutations of the EGFR gene are often associated with well-differentiated adenocarcinoma of the lung. Patients with NSCLC harboring mutations in the EGFR gene are known to respond to EGFR-TKI treatment (3). However, mutations in the downstream signaling effectors of EGFR may result in resistance to EGFR inhibitors. ALK fusion gene-positive lung adenocarcinomas typically occur in young non- or rare smokers. ALK-positive lung adenocarcinoma frequently displays a mucinous cribriform pattern. Crizotinib is a viable option for NSCLC patients harboring ALK rearrangement (4).

Several studies have demonstrated that ALK rearrangement and K-Ras/EGFR mutations are mutually independent and do not coexist in NSCLC $(5,6)$. However, it was recently discovered that ALK rearrangement and EGFR mutation may coexist in the same tumor, with an incidence rate of $1-6 \%(7,8)$. Clinical studies have demonstrated that NSCLC patients with EGFR gene mutations and ALK fusion gene may benefit from EGFRTKIs and ALK inhibitors, respectively. There remains the issue of how to optimize targeted treatment in patients harboring EGFR gene mutations coexisting with ALK-positive NSCLC. EGFR-TKIs are the first-line treatment of advanced NSCLC patients with EGFR mutations alone, with an effectiveness rate of $70-80 \%$ (9) and a progression-free survival time of 9-14 months (10). However, the sensitivity to EGFR-TKIs in patients with coexisting mutations is inconsistent $(7,11)$. ALK rearrangement may be a mechanism mediating primary resistance to EGFR-TKIs. ALK rearrangement is a predictive biomarker of the efficacy of crizotinib. The objective response rate for patients with simple ALK rearrangements receiving crizotinib was reported to be $65.3 \%$; however, the sensitivity of patients with coexisting mutations to ALK inhibitors remains unclear. It was reported that, when patients with coexisting mutations develop disease progression after receiving EGFR-TKI treatment, crizotinib treatment may be replaced with ALK inhibitors $(7,11,12)$. However, an in vitro study demonstrated that aberrant activation of the EGFR signaling pathway is one of the mechanisms mediating tumor cell resistance to ALK inhibitors (8). The results have been conflicting, and there is a need to further investigate the sensitivity of NSCLC patients with coexisting mutations to ALK inhibitors. In addition, the patient's blood type was $\mathrm{AB}, \mathrm{RhD}^{+}$; further investigation may be required to determine whether there is an association between cancer with coexisting gene mutations and blood type. The present case report may provide further patient information, which may help with multi-angle research and disease treatment.

We recommend that the biological behavior of cancers with coexisting genetic mutations and their clinicopathological characteristics are more closely investigated in future studies, in order to determine the sensitivity of such cancers to targeted drugs and select the optimal agent.

\section{References}

1. Lemjabbar-Alaoui H, Hassan OU, Yang YW and Buchanan P: Lung cancer: Biology and treatment options.Biochim Biophys Acta 1856: 189-210, 2015.

2. Yang Y, Song X, Zhu J and Zhou X: A rare case of adenocarcinoma of the lung harboring EGFR, BRAF, and ALK activating mutations. Chest 146 (4_MeetingAbstracts): 640A, 2014.

3. Maemondo M, Inoue A, Kobayashi K, Sugawara S, Oizumi S, Isobe H, Gemma A, Harada M, Yoshizawa H, Kinoshita I, et al: Gefitinib or chemotherapy for non-small-cell lung cancer with mutated EGFR. N Engl J Med 362: 2380-2388, 2010.

4. Shaw AT, Yeap BY, Mino-Kenudson M, Digumarthy SR, Costa DB, Heist RS, Solomon B, Stubbs H, Admane S, McDermott $\mathrm{U}$, el at: Clinical features and outcome of patients with non-small-cell lung cancer who harbor EML4-ALK. J Clin Oncol 27: 4247-4253, 2009.

5. Soda M, Choi YL, Enomoto M, Takada S, Yamashita Y, Ishikawa S, Fujiwara S, Watanabe H, Kurashina K, Hatanaka $\mathrm{H}$, et al: Identification of the transforming EML4-ALK fusion gene in non-small-cell lung cancer. Nature 448: 561-566, 2007. 
6. Li Y, Li Y, Yang T, Wei S, Wang J, Wang M, Wang Y, Zhou Q, Liu $\mathrm{H}$ and Chen J: Clinical significance of EML4-ALK fusion gene and association with EGFR and KRAS gene mutations in 208 Chinese patients with non-small cell lung cancer. PLoS One 8: e52093, 2013.

7. Lee JK, Kim TM, Koh Y,Lee SH, Kim DW, Jeon YK, Chung DH, Yang SC, Kim YT, Kim YW, et al: Differential sensitivities to tyrosine kinase inhibitors in NSCLC harboring EGFR mutation and ALK translocation. Lung Cancer 77: 460-463, 2012.

8. Sasaki T, Koivunen J, Ogino A, Yanagita M, Nikiforow S, Zheng W, Lathan C, Marcoux JP, Du J and Okuda K: A novel ALK secondary mutation and EGFR signaling cause resistance to ALK kinase inhibitors. Cancer Res 71: 6051-6060, 2011.

9. Wu JY, Wu SG, Yang CH, Chang YL, Chang YC, Hsu YC, Shih JY and Yang PC: Comparison of gefitinib and erlotinib in advanced NSCLC and the effect of EGFR mutations. Lung Cancer 72: 205-212, 2011.
10. Mitsudomi T, Morita S, Yatabe Y, Negoro S, Okamoto I, Tsurutani J, Seto T, Satouchi M, Tada H and Hirashima T: Gefitinib versus cisplatin plus docetaxel in patients with non-small-cell lung cancer harbouring mutations of the epidermal growth factor receptor (WJTOG3405): An open label, randomised phase 3 triaL. Lancet Oncol 11: 121-128, 2010.

11. Chen X, Zhang J, Hu Q, Li X and Zhou C: A case of lung adenocarcinoma harboring exon 19 EGFR deletion and EML4-ALK fusion gene. Lung Cancer 81: 308-310, 2013.

12. Miyanaga A, Shimizu K, Noro R, Seike M, Kitamura K, Kosaihira S, Minegishi Y, Shukuya T, Yoshimura A, Kawamoto M, et al: Activity of EGFR-tyrosine kinase and ALK inhibitors for EML4-ALK-rearranged non-small-cell lung cancer harbored coexisting EGFR mutation. BMC Cancer 13: 262, 2013. 